\title{
La fonction \\ biliaire \\ et sa régulation
}

> La fonction biliaire conditionne l'absorption intestinale des lipides, assure l'homéostasie du cholestérol et l'élimination de différents produits de dégradation, comme ceux de l'hémoglobine, sous forme de bilirubine. La bile est élaborée initialement dans les cellules parenchymateuses hépatiques (hépatocytes), puis modifiée par les activités de sécrétion et de réabsorption des cholangiocytes (cellules épithéliales biliaires). Le principal déterminant de la formation de la bile est un processus de filtration osmotique dû au transport actif des acides biliaires et de solutés osmotiquement actifs. Les transporteurs membranaires assurant la formation de la bile sont maintenant en grande partie identifiés. L'expression de ces transporteurs membranaires est régulée par des mécanismes, en particulier transcriptionnels, sous le contrôle de récepteurs nucléaires activés par des ligands dont les principaux sont les acides biliaires, stéroïdes synthétisés dans l'hépatocyte à partir du cholestérol. Les maladies biliaires monogéniques décrites au cours des dernières années illustrent le rôle crucial des transporteurs membranaires dans la fonction biliaire. Les maladies biliaires évoluent vers la cirrhose. Au cours de ces maladies, les acides biliaires modulent l'expression des gènes contrôlant les transporteurs biliaires. Cette régulation peut être considérée comme une réponse adaptative, pouvant contribuer à la variabilité phénotypique de ces maladies. Le traitement de ces affections repose actuellement sur l'administration d'acide ursodésoxycholique et sur la transplantation hépatique, en cas d'échec du traitement médical. Les progrès récents devraient aboutir à la mise au point de nouveaux médicaments ciblés sur les anomalies moléculaires associées à ces entités pathologiques.<

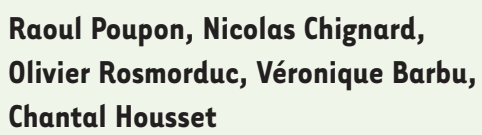

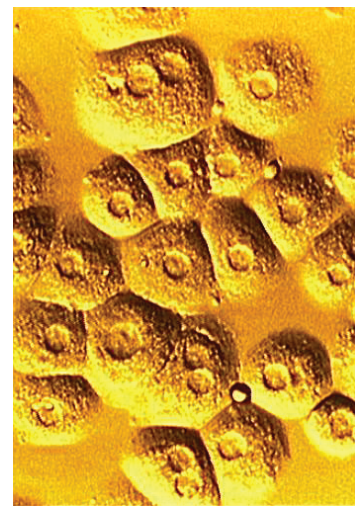

Par l'intermédiaire de la sécrétion biliaire, le foie assure l'élimination de métabolites endogènes ou exogènes, potentiellement toxiques pour l'organisme, et la sécrétion des acides biliaires nécessaires à l'absorption digestive des graisses. Principaux constituants de la bile, les acides biliaires sont synthétisés dans les hépatocytes à partir du cholestérol. Au cours du cycle entéro-hépatique, les acides biliaires sont réabsorbés par les entérocytes de l'iléon terminal et captés à nouveau par les hépatocytes. Les hépatocytes sont des cellules épithéliales polarisées dont le pôle luminal délimite un espace clos appelé canalicule biliaire. La bile est formée dans les canalicules biliaires par un mécanisme de filtration osmotique consécutif au transport actif des acides biliaires et d'autres solutés. La bile parvient au tube digestif par l'intermédiaire de canaux biliaires bordés par les cellules épithéliales biliaires, ou cholangiocytes. Ces derniers participent également à la formation de la bile, essentiellement par l'intermédiaire d'une sécrétion hydro-électrolytique. La plupart des transporteurs hépatocytaires, cholangiocytaires ou entérocytaires impliqués dans la sécrétion biliaire ont été identifiés au cours des dernières années, ainsi que plusieurs voies de régulation de cette sécrétion, notamment par les récepteurs nucléaires des acides biliaires. Ces découvertes récentes ont entraîné une véritable renaissance de la physiologie biliaire. Elles ont également de nombreuses applications dans le domaine des maladies biliaires liées à des anomalies congénitales ou acquises de la sécrétion biliaire.

\section{Les transporteurs hépatobiliaires}

La Figure 1 représente les principaux transporteurs assurant la fonction biliaire [1]. Certains de ces transporteurs de la famille des solute carriers (SLC) sont localisés dans la membrane sinusoïdale des hépatocytes. Ils 
comprennent le NTCP ( $\mathrm{Na}^{+}$-taurocholate cotransporting polypeptide), qui assure la capture hépatocytaire des acides biliaires, et différents membres de la famille des OATP (organic-anion-transporting polypeptides). L'exportation vers l'espace canaliculaire est assurée par plusieurs transporteurs de la membrane canaliculaire appartenant à la superfamille ABC (ATP binding cassette). Ces transporteurs requièrent de l'énergie, fournie par l'hydrolyse de I'ATP. Les principaux transporteurs ABC sont MDRI (multidrugresistance-1 $P$-glycoprotein), qui transporte les cations organiques (colchicine, ciclosporine, doxorubicine, tamoxifène, tacrolimus...), BSEP (bile salt export pump), principal transporteur des acides biliaires, MRP2 (multidrug-resistance-associated protein 2), responsable du transport de la bilirubine conjuguée et du glutathion, MDR3 (multidrug-resistance 3 Pglycoprotein), responsable du transport des phospholipides, et l'hétérodimère ABCG5-ABCG8 (ATP-binding cassette, sub-family G, member 5 ou 8), responsable du transport du cholestérol. Certains transporteurs des acides biliaires et des solutés organiques sont également présents dans les cholangiocytes, en particulier ASBT (apical sodium-dependent bile salt transporter). Ce transporteur a été initialement identifié dans la membrane apicale des cellules épithéliales de l'intestin grêle terminal où il assure la

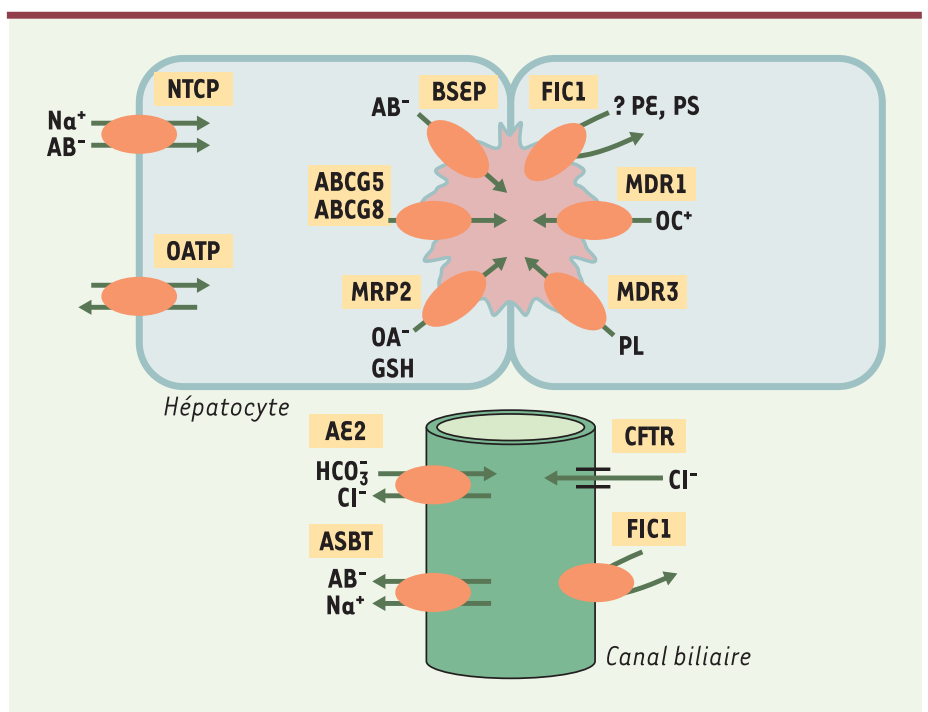

Figure 1. Localisation des principaux transporteurs hépatobiliaires. Dans les hépatocytes, ces transporteurs sont localisés, d'une part, dans la membrane sinusoïdale (NTCP: $\mathrm{Na}^{+}$-taurocholate cotransporting polypeptide; OATP: organic-anion-transporting polypeptides) et, d'autre part, dans la membrane canaliculaire, où ils assurent l'exportation des acides biliaires $\left(A B^{-}\right)$et d'autres composés vers l'espace canaliculaire (en rose). BSEP: bile salt export pump; MDRI: multidrug-resistance-1 P-glycoprotein; MRP2: multidrug-resistance-associated protein 2; MDR3: multidrug-resistance-3 P-glycoprotein; ABCG5: ATP-binding cassette, sub-family G (WHITE), member 5 (sterolin 1); ABCG8: ATP-binding cassette, sub-family G (WHITE), member 8 (sterolin 2); FICl: familial intrahepatic cholestasis 1; PE: phosphatidyléthanolamine; PS: phosphatidylsérine; $\mathrm{OC}^{+}$: cations organiques; PL: phospholipides; $\mathrm{OA}^{-}$: anions organiques; GSH: glutathion. Seule la membrane apicale des cholangiocytes, qui délimite la lumière du canal biliaire, est représentée (en vert). Aع2: anion exchanger isoform 2; ASBT: apical sodium-dependent bile salt transporter; CFTR: cystic fibrosis transmembrane conductance regulator. réabsorption active des acides biliaires au cours du cycle entéro-hépatique. Dans les cholangiocytes, sa fonction serait de réabsorber les acides biliaires de la bile en amont d'un obstacle sur les voies biliaires. MDRl est également présent dans la membrane apicale des cholangiocytes. Deux transporteurs, CFTR (cystic fibrosis transmembrane conductance regulator) et AE2 (anion exchanger isoform 2), situés dans la membrane apicale des cholangiocytes, ont pour fonction d'assurer la sécrétion biliaire des bicarbonates.

Toutes ces protéines, exprimées de façon constitutive par l'hépatocyte ou les cholangiocytes, subissent une régulation, en particulier transcriptionnelle, sous le contrôle de récepteurs nucléaires dont les ligands sont les acides biliaires, les oxystérols, la vitamine $A$ et ses dérivés, et les xénobiotiques.

\section{Régulation par les récepteurs nucléaires}

Les principaux récepteurs nucléaires impliqués dans I'homéostasie du cholestérol et des acides biliaires, et dans l'élimination des xénobiotiques, sont le FXR (farnesoid $X$ receptor), le PXR (pregnane $X$ receptor), isolé chez la souris, dont l'homologue humain est le SXR (steroid and xenobiotic receptor), le CAR (constitutive androstane receptor) et le LXR $\alpha$ et $\beta$ (liver $X$ receptor) [2].

FXR a pour ligands les acides biliaires, en particulier l'acide chénodésoxycholique. La Figure 2 schématise le rôle de FXR dans l'homéostasie des acides biliaires. La liaison de FXR à son ligand entraîne une diminution de l'expression du NTCP et de CYP7Al et CYP8B1, qui codent respectivement pour la $7 \alpha$ - et la $12 \alpha$-hydroxylase responsables, dans l'hépatocyte, de la synthèse des acides biliaires à partir du cholestérol. FXR entraîne également une augmentation de la transcription des gènes codant pour les protéines BSEP, MRP2 et MDR3. FXR est donc un régulateur majeur de la concentration intra-hépatocytaire des acides biliaires. La fonction de FXR a pu être précisée à l'aide de souris dont le gène codant pour ce facteur de transcription a été invalidé. Il existe, chez ces souris, une accumulation hépatique et sanguine d'acides biliaires. Cette accumulation s'accentue avec l'âge et entraîne progressivement une atteinte hépatique qui devient sévère si les souris reçoivent un régime enrichi en acide cholique [3]. SXR/PXR, quant à eux, ont pour principaux ligands certains xénobiotiques, en particulier la rifampicine et les acides biliaires hydrophobes comme le lithocholate. Les principaux gènes cibles de PXR sont CYP3A4 et MRP2. Le CYP3A4 est responsable de l'hydroxylation - et donc de 
l'élimination -des acides biliaires hydrophobes. Les souris dont le gène $P X R$ a été invalidé sont incapables de métaboliser nombre de médicaments et développent une atteinte hépatique sévère en raison d'un défaut de détoxification de l'acide lithocholique. PXR, comme FXR, joue donc un rôle physiologique protecteur vis-à-vis de l'excès d'acides biliaires dans les cellules hépa-

tiques $[4,5]$.

Quant à CAR, il est un récepteur des xénobiotiques dont les principales fonctions sont de régler le métabolisme de la bilirubine et des xénobiotiques. Les principaux gènes cibles hépatocytaires de CAR sont ceux codant pour les cytochromes P450, 2B, $2 C, 3 A$ et $4 A$. CAR induit par ailleurs l'expression de l'ensemble des protéines impliquées dans la clairance de la bilirubine. CAR est activé par des taux élevés de bilirubine intra-hépatocytaire. Autrement dit, la bilirubine induit sa propre clairance. L'induction du cytochrome P450 par CAR est en principe une réponse protectrice vis-à-vis de certains xénobiotiques [5].

Enfin, LXR constitue une classe de récepteurs nucléaires activés par les oxystérols. LXR $\alpha$ et $\beta$ règlent l'expression des gènes impliqués dans l'homéostasie lipidique. LXR est exprimé non seulement dans le foie, mais également dans d'autres tissus, en particulier dans les macrophages, où les oxystérols sont présents du fait d'une internalisation des LDL (low density lipoproteins) oxydées ou d'une production in situ à partir du cholestérol. Les gènes hépatiques cibles de LXR sont CYP7A1, ABCG5, ABCG8 (facteurs impliqués dans le transport du cholestérol de l'hépatocyte vers le canalicule, dans la régulation de l'absorption digestive du cholestérol et dans l'efflux du cholestérol des macrophages). Les souris dont le gène $L X R$ a été invalidé ( $L X R \alpha$ et $L X R \beta$ ), chez lesquelles le foie accumule du cholestérol, peuvent développer une cirrhose par un mécanisme encore mal compris [6].

\section{Physiopathologie des maladies biliaires}

Les maladies biliaires sont associées à des anomalies de sécrétion des hépatocytes ou des cholangiocytes. Les mutations de plusieurs transporteurs $A B C$ sont responsables de cholestases familiales progressives (PFIC) chez l'enfant [7]. Ainsi, les mutations d'un gène codant pour la protéine $\mathrm{FICl}$ (familial intrahepatic cholestasis 1), présente dans la membrane canaliculaire et dans les cholangiocytes, mais aussi dans le pancréas et l'intestin, sont responsables de la maladie de Byler. Ce gène n'a pas été cité ci-dessus car le rôle physiologique de $\mathrm{FICl}$, une protéine responsable de la translocation de la phosphatidyléthanolamine et de la phosphatidylsérine dans les membranes, est encore mal connu. La cholestase familiale de type 2 est due à des mutations de BSEP. La cholestase familiale de type 3 est en rapport avec des mutations du gène MDR3. Au cours de cette maladie cholestatique, il existe une inflammation et une altération des petites voies biliaires, comme au cours de la cirrhose biliaire primitive.
La bile de ces patients est caractérisée par une concentration très basse en phospholipides. L'étude de ce gène nous a également permis de montrer qu'il était responsable d'une maladie de l'adulte jeune associant une microlithiase biliaire et une cholangiopathie parfois responsable d'atteinte hépatique sévère $[7$, 8]. Les mutations du CFTR sont responsables d'un défaut de sécrétion de chlore par les cholangiocytes [9], avec comme conséquence une cholestase et une inflammation des voies biliaires touchant environ $10 \%$ à $20 \%$ des patients atteints de mucoviscidose. Nous avons rapporté récemment le rôle aggravant potentiel des mutations du CFTR dans certaines formes de maladies inflammatoires des voies biliaires de l'adulte [10]. De plus, des altérations fonctionnelles de ces transporteurs pourraient être responsables de maladies biliaires acquises, notamment d'origine médicamenteuse [11].

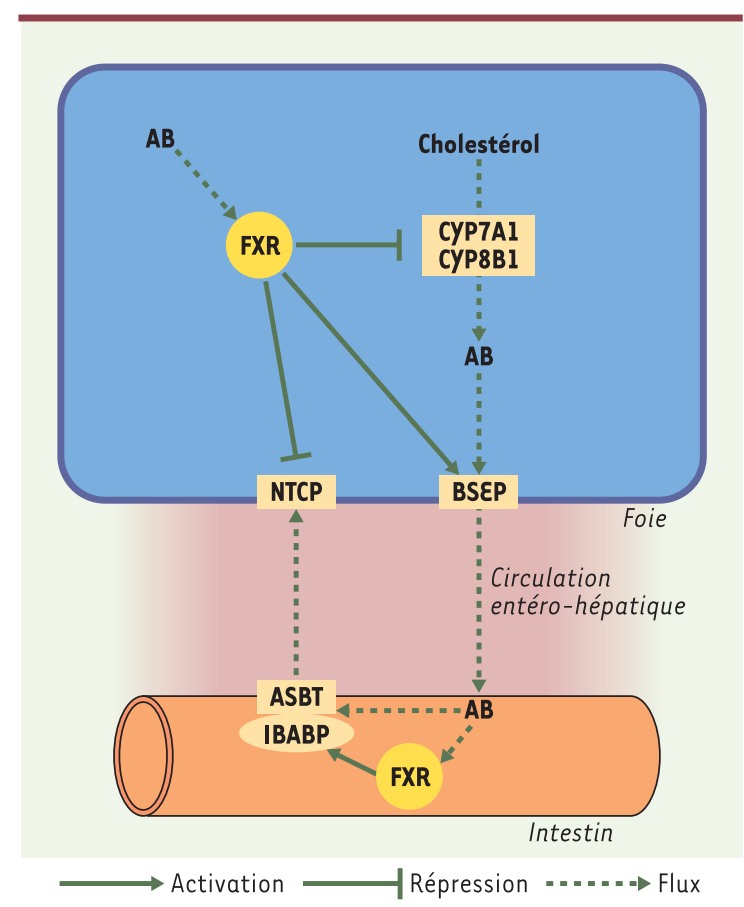

Figure 2. Régulation de l'homéostasie biliaire par le récepteur nucléaire FXR. Dans l'hépatocyte, la liaison de FXR à son ligand, l'acide biliaire $(A B)$, entraîne une régulation transcriptionnelle négative de NTCP ( $\mathrm{Na}^{+}$-taurocholate cotransporting polypeptide) et de CYP7Al et CYP8B1, enzymes responsables de la synthèse des acides biliaires à partir du cholestérol, et une régulation transcriptionnelle positive de BSEP (bile salt export pump). Dans l'entérocyte, la liaison de FXR à l'acide biliaire $(A B)$ entraîne une régulation transcriptionnelle positive d'IBABP (ileal bile acid-binding protein), partenaire intracytoplamique d'ASBT (apical-sodium dependent bile salt transporter), nécessaire à l'absorption intestinale des acides biliaires par ASBT. Le résultat de la régulation par FXR est donc une diminution de l'accumulation des acides biliaires dans l'hépatocyte et dans la lumière intestinale. 


\section{Régulation et adaptation des transporteurs hépatobiliaires dans les cholestases expérimentales ou acquises chez l'homme}

Il existe, au cours des maladies cholestatiques, une modification d'expression des transporteurs biliaires qui peut être considérée comme un mécanisme de protection [12-15]. S'agissant des transporteurs basolatéraux, on observe, dans toutes les formes de cholestase, une diminution de l'expression de NTCP, aboutissant à une diminution de la capture des acides biliaires. S'agissant des transporteurs canaliculaires, il a été montré que l'expression de MDRl est augmentée au cours des maladies cholestatiques. Dans les modèles expérimentaux, l'expression hépatique de MDR3 est augmentée, alors que l'expression de BSEP et de MRP2 est peu modifiée. S'agissant des transporteurs intestinaux, des études préliminaires indiquent que l'expression de MRP2 est augmentée et celle de MDR1 diminuée dans l'intestin après cholestase par ligature-section de la voie biliaire principale chez le rat. Les modifications d'ASBT, qui assure la réabsorption des acides biliaires, sont controversées, mais les acides biliaires augmentent l'expression d'IBAP (ileal bile acid-binding protein), protéine de liaison des acides biliaires à l'intérieur de l'entérocyte.

Les mécanismes de la régulation adaptative des transporteurs biliaires constituent une thématique émergente dont l'intérêt est évident puisque leur connaissance ouvre la voie à des thérapeutiques médicales adaptées à chaque situation. Les agonistes de la protéine FXR augmentent l'expression de BSEP, MRP2 et $M D R 3$ et ont, d'ores et déjà, fait la preuve de leur efficacité dans différents modèles expérimentaux de cholestase [12]. L'acide ursodésoxycholique et la rifampicine, des ligands de SXR, ont un effet bénéfique dans les maladies cholestatiques, suggérant que ce facteur de transcription ou ses modifications pourraient jouer un rôle dans l'expression clinique de ces maladies.

\section{Conclusions}

La connaissance actuelle des voies métaboliques et des transporteurs dont dépend la sécrétion biliaire permet de mieux comprendre la physiopathologie des maladies biliaires génétiques ou acquises. La régulation par différents récepteurs nucléaires ayant pour ligands les acides biliaires est au centre de mécanismes d'adaptation qui protègent l'organisme des effets délétères de la cholestase. Les stratégies thérapeutiques actuellement envisagées visent à stimuler ces mécanismes d'adaptation qui, spontanément, sont insuffisants pour empêcher l'évolution des maladies biliaires. $\diamond$

\section{SUMMARY}

\section{Biliary function and its regulation}

Biliary function is essential for intestinal absorption of fat, homeostasis of cholesterol and elimination of diverse metabo- lic end-products. Bile is elaborated in hepatocyte canaliculi and modified by cholangiocytes through both secretion and absorption processes. The main determinant of bile formation is an osmotic filtration process resulting from active transport of bile acids and other osmotic solutes. Most of the membrane transporters ensuring bile formation have now been identified. The expression of these membrane transporters is regulated in particular through transcriptional mechanisms under the control of nuclear receptors activated by ligands, such as bile acids, which act as endogenous steroids synthesized from cholesterol in hepatocytes. Monogenic cholestatic diseases illustrate the key role of membrane transporters in biliary function. Bile acids are potent modulators of transporters and thus trigger an adaptative response to cholestasis. The extent of this adaptative response could explain the compelling phenotypic variability of cholestatic diseases in childhood and adults. The firstline medical treatment is currently ursodeoxycholic acid. In case of failure of this medical treatment, liver transplantation is required. Recent progress in the molecular pathogenesis of bile formation and cholestatic liver diseases is expected to provide the design for drugs targeted to the molecular abnormalities responsible of cholestatic diseases. $\diamond$

\section{RÉFÉRENCES}

1. Jansen PL. From classic bile physiology to cloned transporters. Semin Liver Dis $2000 ; 20: 245-50$.

2. Karpen SJ. Nuclear receptor regulation of hepatic function. J Hepatol 2002; 36 : $832-50$.

3. Sinal CJ, Tohkin M, Miyata M, et al. Targeted disruption of the nuclear receptor FXR/BAR impairs bile acid and lipid homeostasis. Cell 2000; 102: 731-44.

4. Xie W, Radominska-Pandya A, Shi Y, et al. An essential role for nuclear receptors SXR/PXR in detoxification of cholestatic bile acids. Proc Natl Acad Sci USA 2001 98: 3375-80.

5. Xie W, Yeuh MF, Radominska-Pandya A, et al. Control of steroid, heme, and carcinogen metabolism by nuclear pregnane $X$ receptor and constitutive androstane receptor. Proc Natl Acad Sci USA 2003; 100: 4150-55.

6. Peet DJ, Turley SD, Ma W, et al. Cholesterol and bile acid metabolism are impaired in mice lacking the nuclear oxysterol receptor LXR alpha. Cell 1998; 93: 693-704.

7. Jacquemin $\varepsilon$, Hadchouel M. Genetic basis of progressive familial intrahepatic cholestasis. J Hepatol 1999; 31: 377-81.

8. Rosmorduc 0 , Hermelin B, Boelle Py, et al. ABCB4 gene mutation associated cholelithiasis in adults. Gastroenterology 2003; 125: 452-9.

9. Dray-Charier N, Paul A, Scoazec Jy, et al. Expression of delta F508 cystic fibrosis transmembrane conductance regulator protein and related chloride transport properties in the gallbladder epithelium from cystic fibrosis patients. Hepatology $1999 ; 29: 1624-34$

10. Girodon $\varepsilon$, Sternberg D, Chazouilleres 0 , et al. Cystic fibrosis transmembrane conductance regulator (CFTR) gene defects in patients with sclerosing cholangitis. J Hepatol 2002; 37: 192-7.

11. Fouassier L, Kinnman N, Lefevre B, et al. Contribution of mrp2 in alterations of canalicular bile formation by the endothelin antagonist bosentan. J Hepatol 2002 ; 37: 184-91.

12. Liu Y, Binz J, Numerick MJ, et al. Hepatoprotection by the farnesoid X receptor agonist GW4064 in rat models of intra- and extrahepatic cholestasis. J Clin Invest 2003; 112: 1678-87.

13. Lee J, Boyer JL. Molecular alterations in hepatocyte transport mechanisms in acquired cholestatic liver disorders. Semin Liver Dis 2000; 20:373-84.

14. Lee J, Azzaroli F, Wang L, et al. Adaptive regulation of bile salt transporters in kidney and liver in obstructive cholestasis in the rat. Gastroenterology 2001; 121 : 1473-84.

15. Kojima H, Nies AT, Konig J, et al. Changes in the expression and localization of hepatocellular transporters and radixin in primary biliary cirrhosis. J Hepatol 2003; 39 $3702-5$
TIRÉS À PART

R. Poupon 